\section{Avaliação participativa do Programa Nacional de Suplementação de Vitamina $A$ em um município da Região Nordeste do Brasil}

\author{
Participatory evaluation of the National Program for \\ Vitamin A Supplementation in a municipality in \\ Northeast Brazil
}

\author{
${ }^{1}$ Centro de Ciências da \\ Saúde, Universidade Federal \\ da Paraíba, João Pessoa, \\ Brasil. \\ 2 Coordenação Geral da \\ Política de Alimentação \\ e Nutrição, Ministério da \\ Saúde, Brasília, Brasil. \\ 3 Organização Pan- \\ Americana da Saúde, \\ Brasília, Brasil. \\ Correspondência \\ E. R. Almeida \\ Centro de Ciências da Saúde, \\ Universidade Federal da \\ Paraíba. \\ Av. Sinésio Guimarães 491, \\ João Pessoa, $P B$ \\ 58040-400, Brasil. \\ erikinhanutri@hotmail.com
}

\begin{abstract}
This study aimed to evaluate the National Program for Vitamin A Supplementation in a municipality (county) in Northeast Brazil, with participation by the parents or guardians of children under five years of age. This was a cross-sectional, population-based study using both qualitative and quantitative approaches. A total of 657 interviews were performed, and the vitamin A doses recorded on the children's immunization cards were analyzed. The population has access to high doses of vitamin A. However, knowledge of the vitamin A supplementation program was limited, especially among parents with less schooling. The program's coverage fell short of the Ministry of Health's target, especially after 18 months of age. It is thus necessary to intensify the program's educational activities in order to raise awareness among families of under-five children on the importance of vitamin A, its supplementation, and the importance of eating natural sources of this nutrient for the child's health.
\end{abstract}

Vitamin A; Nutrition Programs and Policies; Child Health
Erika Rodrigues de Almeida 1

Alice Teles de Carvalho 1

Eduardo Augusto Fernando Nilson 2

Janine Giuberti Coutinho 3

Juliana Amorim Ubarana 2

\section{Introdução}

A carência de vitamina A é uma importante deficiência nutricional em todo o mundo, sendo considerada um problema de saúde pública em vários países em desenvolvimento, inclusive o Brasil 1.

Desde o início do século XX, são realizadas pesquisas que abordam a deficiência de vitamina A e suas complicações. Durante as décadas de 80 e 90, estudos sobre essa temática abordavam, em geral, a epidemiologia desse agravo 2,3. Nesse mesmo período, ainda foram realizados estudos de metanálises que encontraram efeito positivo da suplementação de vitamina A na redução da morbimortalidade infantil 4,5 . Estudos mais recentes reafirmam a redução da severidade de episódios de diarréia por meio da suplementação com vitamina A 6,7 .

Devido à associação entre vitamina A e morbimortalidade materno-infantil, os diversos organismos internacionais estimularam programas de prevenção desta deficiência, que incluem a suplementação da vitamina. O Brasil iniciou as ações de suplementação de vitamina A na década de 80, integradas ao Programa Nacional de Imunização. Em 1994, essas ações foram institucionalizadas com a criação do Programa Nacional de Controle das Deficiências de Vitamina A, no âmbito do Instituto Nacional de Alimentação e Nutrição 8,9 . 
No desenho atual, o programa recomenda a distribuição de cápsulas de megadose de vitamina A para crianças de 6 a 59 meses de idade e mulheres no pós-parto imediato, além do provimento de ações educativas em alimentação e nutrição que estimulem a adoção de hábitos alimentares saudáveis. Essas ações são desenvolvidas em regiões consideradas de risco para essa deficiência (Região Nordeste, norte de Minas Gerais, Vale do Jequitinhonha, Vale do Mucurici e Vale do Ribeira em São Paulo), e devem ser realizadas na rotina dos serviços de atenção primária à saúde, bem como nas campanhas de multivacinação, pelas equipes de saúde da família 10,11.

Nas regiões consideradas endêmicas para a deficiência dessa vitamina, a meta para cobertura pelo Programa Nacional de Suplementação de Vitamina A (PNVITA) é de $100 \%$ para crianças de 6 a 11 meses de idade. Para as crianças de 12 a 59 meses, a meta é de $100 \%$ para a primeira megadose e de $60 \%$ para a segunda 12 . Nesse último caso, a perspectiva da Coordenação Geral da Política de Alimentação e Nutrição (CGPAN) do Ministério da Saúde é o aumento gradativo dessa meta, a partir do fortalecimento de estratégias de educação, melhoria de gestão, sensibilização de profissionais, gestores e população participante do programa.

Apesar da existência de estudos sobre a prevalência da deficiência de vitamina A, suas complicações e sobre a eficácia da suplementação na redução da morbimortalidade materno-infantil $13,14,15$, poucos são os que avaliam o processo de operacionalização do PNVITA com a participação do público-alvo. Merece destaque o estudo de Martins et al. 16, que usando um enfoque multidimensional avaliou o referido programa no Estado da Bahia, envolvendo os responsáveis pelas crianças participantes do programa.

A literatura especializada reconhece que as atividades de avaliação propiciam o comprometimento e a responsabilidade de atores em torno de objetivos a serem partilhados e conjugadamente alcançados. Programas sociais, por exemplo, precisam ser efetivos e cumprir satisfatoriamente as metas propostas para que sejam eficientes do ponto de vista da utilização consistente de recursos, diante das medidas de efetividade alcançadas 17 .

De acordo com Minayo \& Cruz Neto 18, o processo avaliativo participativo, além de promover a integração objetiva e subjetiva, inclui os atores sociais do programa não só como objetos de análise, mas também como sujeitos de auto-avaliação, o que possibilita que durante o processo eles se apropriem da compreensão dos dados, com o intuito de modificarem e melhorarem as ações.
Desde 2003, o Ministério da Saúde do Brasil vem desenvolvendo ações no sentido de institucionalizar o processo de avaliação no âmbito da atenção primária à saúde 19 . Nesse contexto, a CGPAN aderiu à proposta avaliativa de projeto desenvolvido pela Organização das Nações Unidas para Agricultura e Alimentação (FAO) em parceria com quatro países (Argentina, Brasil, México e Panamá), intitulado Análise e Fortalecimento de Programas de Alimentação e Nutrição Comunitária.

No Brasil, a pesquisa incluiu a análise do PNVITA em município da Região Nordeste do país, visando a identificar as possíveis limitações e dificuldades em sua operacionalização, assim como as potencialidades, para traçar um plano de ação na perspectiva de aperfeiçoamento. Para tanto, foram aplicadas diferentes metodologias e investigadas as percepções de diversos atores sociais sobre o funcionamento do programa.

O presente trabalho apresenta os resultados referentes à pesquisa avaliativa participativa realizada no Município de Cabedelo, Estado da Paraíba, que teve como objetivo analisar o funcionamento do PNVITA sob a perspectiva dos responsáveis por crianças menores de cinco anos.

\section{Material e métodos}

\section{Desenho do estudo e cálculo da amostra}

Realizou-se uma pesquisa avaliativa participativa, utilizando o desenho de um estudo epidemiológico transversal, com base populacional. A população foi composta de responsáveis por crianças menores de cinco anos, residentes no Município de Cabedelo, que compareceram aos postos de vacinação na segunda etapa da Campanha de Multivacinação de 2008.

Os critérios de inclusão no estudo foram: pai ou mãe, independentemente da idade, ou outro responsável com idade igual ou superior a 18 anos, que concordasse em participar da pesquisa, mediante assinatura de Termo de Consentimento Livre e Esclarecido.

Optou-se por entrevistar os responsáveis partindo da lógica de que estes representam a inserção da criança no PNVITA e, ainda, que o acesso da criança às ações relacionadas ao programa depende do conhecimento de seus responsáveis acerca deste, de sua sensibilização quanto à importância do mesmo para a saúde da criança e de sua co-responsabilização no alcance dos objetivos propostos pelo programa.

A amostragem da pesquisa foi do tipo probabilística por conglomerado, com duas etapas de seleção. A primeira etapa compreendeu o sorteio 
aleatório de 15 das 19 unidades de saúde da família (USF), que também funcionam como postos de vacinação (os conglomerados). Em seguida, com base na previsão do número de crianças a serem imunizadas em cada posto de vacinação do município, de acordo com a primeira etapa da Campanha de Multivacinação de 2008, estimou-se o número de responsáveis a serem entrevistados. Como o número de responsáveis não é registrado nas campanhas de multivacinação, mas sim o número de crianças imunizadas, considerou-se um responsável para cada duas crianças a serem vacinadas.

Para que a amostra fosse representativa, decidiu-se trabalhar com aproximadamente $70 \%$ da demanda prevista em cada posto. Foi realizada uma amostragem sistemática, adotando-se o critério de entrevistar seqüencialmente dois responsáveis sim e um não, por ordem de chegada ao posto de vacinação. Como o percentual de entrevistas inicialmente previsto foi extrapolado, foi necessário equilibrar o total de entrevistas realizadas em cada USF, de acordo com a representatividade percentual no total da amostra do município, utilizando-se um fator de expansão.

\section{Coleta de dados}

Os dados foram coletados por meio de entrevista estruturada, usando-se um questionário contendo questões abertas e fechadas, elaborado com base nos documentos oficiais que normatizam o programa na literatura científica da área, nas discussões com representantes da CGPAN e da Ação Brasileira pela Nutrição e Direitos Humanos (ABRANDH).

No intuito de testar a adequação do questionário elaborado, assim como da metodologia de coleta de dados proposta (entrevista estruturada), foi realizado um teste-piloto em duas USF do Município de João Pessoa, Paraíba. Realizou-se uma visita em cada unidade, sendo aplicados em torno de vinte questionários em cada uma.

Durante a realização do teste-piloto observou-se o tempo médio gasto em cada entrevista e, principalmente, se a disposição das perguntas no questionário estava coerente, considerando a possibilidade de reordenamento destas quando da observância de indução de respostas com base em questões precedentes.

O teste-piloto também permitiu alterar a forma de redação de algumas questões, em virtude destas não terem sido facilmente compreendidas pelos entrevistados, sendo necessárias maiores explicações acerca do questionamento realizado.

As perguntas incluídas no questionário abordavam o acesso às ações e serviços de saúde e nu- trição, o conhecimento acerca do PNVITA e sobre a vitamina A (importância, agravos decorrentes da carência vitamínica, fontes alimentares). Além disso, dispunha de campo para registro das doses de vitamina A apontadas nos cartões de vacinação das crianças, com o objetivo de avaliar a cobertura do programa para as crianças de 6 a 59 meses de idade.

Foram investigadas as variáveis exploratórias como o grau de parentesco do entrevistado com a criança - mãe, pai, avó(ô), tia(o), irmã(ão) e outro -; a raça/cor autodeclarada do responsável (branca, parda/mulata/morena, negra/preta e amarela/ocidental); a escolaridade, em anos de estudo; a idade do entrevistado e a idade da criança.

Para a realização das entrevistas foram selecionados e treinados 34 alunos do curso de graduação em Nutrição da Universidade Federal da Paraíba (UFPB). Para acompanhar a coleta de dados foi treinado um coordenador para cada posto de vacinação. Esse convidava os responsáveis por crianças menores de cinco anos a participarem da pesquisa, ainda na sala de espera. Aqueles que se enquadravam nos critérios de inclusão e aceitavam participar da pesquisa eram encaminhados pelo coordenador à sala da entrevista, após suas crianças terem sido vacinadas. Os coordenadores também apoiavam os entrevistadores em caso de dúvidas.

\section{Sistematização e análise dos dados coletados}

Os dados coletados foram armazenados utilizando-se o programa Excel for Windows 2003 (Microsoft Corp., Estados Unidos). A análise estatística dos resultados obtidos foi realizada por meio do programa Epi Info versão 6.04 (Centers for Disease Control and Prevention, Atlanta, Estados Unidos).

As questões fechadas geraram tabelas de freqüência simples. A análise das questões abertas foi realizada nas seguintes etapas: (a) identificouse a idéia central de cada resposta apresentada, baseada no questionamento realizado; (b) criaram-se categorias com base nas idéias extraídas das respostas; (c) codificaram-se as categorias para análise das respostas.

Especificamente para o tópico conhecimento acerca da vitamina $\mathrm{A}$, as respostas foram analisadas em três categorias: coerente, incoerente e genérica (características não específicas da vitamina A).

Para o eixo importância da vitamina A foram consideradas coerentes as respostas com os seguintes conteúdos: redução da gravidade de infecções, atuação no processo de crescimento e desenvolvimento infantis, prevenção de doenças 
relacionadas à visão, atuação na diferenciação celular, manutenção da imunidade, proteção da capacidade funcional dos órgãos de reprodução e relação com a saúde da pele, cabelo e unhas. No eixo agravos decorrentes da deficiência de vitamina A foram considerados coerentes os problemas relacionados à visão; à pele, cabelo e unhas; ao crescimento e desenvolvimento; e à redução da imunidade. Levando-se em conta que a deficiência de vitamina A pode causar um tipo de anemia, as referências relacionadas a esta patologia também foram consideradas coerentes 20.

Os dados foram analisados por meio de cruzamento entre as questões e a variável escolaridade, acompanhado de teste qui-quadrado. Foi utilizado nível de significância de 5\%. Para cada uma das proporções foi calculado o intervalo de 95\% de confiança (IC95\%), segundo os anos de estudo.

Para a análise da cobertura de vitamina A, a idade das crianças foi calculada em dias, considerando-se o dia do nascimento até o dia da coleta de dados (9 de agosto de 2008). Para avaliar se a criança estava com a vitamina A em dia, a referência de adequação foi baseada no número de doses indicadas para cada faixa etária, considerando a suplementação semestral recomendada pelo Ministério da Saúde 10,11 e o número de doses recebidas pela criança.

O estudo foi analisado e aprovado pelo Comitê de Ética em Pesquisa do Centro de Ciências da Saúde da UFPB, sob o protocolo no. 138/08.

\section{Resultados}

Considerando o objetivo do presente trabalho, que é avaliar o PNVITA com a participação dos responsáveis de crianças menores de cinco anos, e sendo a USF local de desenvolvimento das ações relacionadas ao programa para esta população, os resultados seguintes dizem respeito à percepção dos responsáveis que, ao serem entrevistados, referiram o acompanhamento das suas crianças por uma USF.

\section{Características da população estudada}

A população estudada foi de 657 indivíduos, correspondentes em números expandidos a 1.882 responsáveis por crianças menores de cinco anos acompanhadas por USF. Esse quantitativo representa $85,5 \%$ de todos os entrevistados no estudo. Os responsáveis apresentavam, predominantemente, entre $25-34$ anos de idade $(44,4 \%$ da amostra), menos de 8 anos de estudo (56,5\% da amostra) e eram, na sua maioria $(78,3 \%)$, mães de crianças menores de cinco anos que representam a população-alvo do PNVITA.

\section{Conhecimento acerca do PNVITA}

Observou-se que menos da metade dos entrevistados (43,5\%; IC95\%: 41,25-45,75) referiu conhecer ou já ter ouvido falar sobre o PNVITA. Identificou-se, ainda, que com o aumento dos anos de estudo há uma redução na proporção de entrevistados que referiram conhecer o PNVITA. No entanto, esse comportamento se diferencia no grupo de 12 anos e mais de estudo, cuja proporção apresenta similaridade com aquela identificada no grupo de 5-8 anos de estudo (Tabela 1).

Dos entrevistados que afirmaram conhecer o programa, 43,7\% (IC95\%: 41,45-45,95) relataram saber algo a respeito, sendo identificadas proporções mais elevadas nos grupos com maiores anos de estudo. No entanto, mais uma vez, o grupo com maior escolaridade (12 e mais anos de estudo) apresentou comportamento diferenciado, visto que a sua proporção é inferior à observada no grupo de 9-11 anos de estudo (Tabela 1).

Aos entrevistados que afirmaram saber algo a respeito do programa, solicitou-se justificativa aberta sobre o tipo de conhecimento. A análise das respostas gerou as seguintes categorias: funções da vitamina A (28,3\%); administração das cápsulas de vitamina A (13,3\%); atividade educativa $(0,7 \%)$; outros $(1,4 \%)$; não soube identificar (56,3\%).

Menos de 20\% (13,8\%; IC95\%: 12,23-15,37) dos entrevistados que relataram saber algo a respeito do programa afirmaram conhecer as suas atividades, sendo as maiores proporções de conhecimento identificadas nos grupos de 5-8 e 9-11 anos de estudo, os quais apresentam similaridade (Tabela 1). Entre os que afirmaram conhecer as atividades relacionadas ao PNVITA, predominou o percentual de entrevistados que não soube listar tais ações $(86,2 \%)$, seguido das categorias: realização de atividades educativas (6,4\%); administração das cápsulas de vitamina A (4\%); e consultas nas USF $(2,1 \%)$.

No tocante à periodicidade de suplementação das megadoses de vitamina $\mathrm{A}$, observou-se o desconhecimento desta informação pela maioria dos entrevistados, visto que apenas $35,3 \%$ (IC95\%: 33,13-37,47) referiram saber o intervalo de tempo em que suas crianças devem ser suplementadas. Observou-se menor proporção de conhecimento no grupo de menor escolaridade (0-4 anos), o qual se diferencia dos demais quando analisados os intervalos de confiança (Tabela 1).

Entre os que afirmaram saber a periodicidade de suplementação de vitamina A, quase $70 \%$ (68\%; IC95\%: 65,88-70,12) referiram a periodi- 
Conhecimento dos responsáveis acerca do Programa Nacional de Suplementação de Vitamina A (PNVITA). Município de Cabedelo, Paraíba, Brasil, 2008.

\begin{tabular}{|c|c|c|c|c|c|c|}
\hline \multirow[t]{3}{*}{ Questões } & \multicolumn{4}{|c|}{ Escolaridade (anos de estudo) } & \multirow{2}{*}{ Total } & \multirow{3}{*}{$\begin{array}{l}\text { Valor } \\
\text { de p * }\end{array}$} \\
\hline & $0-4$ & $5-8$ & $9-11$ & 12 e mais & & \\
\hline & \% (IC95\%) & \% (IC95\%) & \% (IC95\%) & \% (IC95\%) & $\%(I C 95 \%)$ & \\
\hline Conhece o PNVITA (já ouviu falar) & $\begin{array}{c}52,6 \\
(50,33-54,87)\end{array}$ & $\begin{array}{c}44,9 \\
(42,64-47,16)\end{array}$ & $\begin{array}{c}36,3 \\
(34,12-38,48)\end{array}$ & $\begin{array}{c}41,3 \\
(39,07-43,53)\end{array}$ & $\begin{array}{c}43,5 \\
(41,25-45,75)\end{array}$ & $<0,001$ \\
\hline Referiu saber algo sobre o PNVITA ** & $\begin{array}{c}34,7 \\
(32,54-36,86)\end{array}$ & $\begin{array}{c}38,2 \\
(35,99-40,41)\end{array}$ & $\begin{array}{c}57,1 \\
(54,85-59,35)\end{array}$ & $\begin{array}{c}49,2 \\
(46,93-51,47)\end{array}$ & $\begin{array}{c}43,7 \\
(41,45-45,95)\end{array}$ & $<0,001$ \\
\hline Conhece as atividades do PNVITA ** & $\begin{array}{c}11,5 \\
(10,05-12,95)\end{array}$ & $\begin{array}{c}15,3 \\
(13,67-16,93)\end{array}$ & $\begin{array}{c}15,3 \\
(13,67-16,93)\end{array}$ & $\begin{array}{c}13,0 \\
(11,47-14,53)\end{array}$ & $\begin{array}{c}13,8 \\
(12,23-15,37)\end{array}$ & 0,003 \\
\hline Referiu conhecer a periodicidade de suplementação & $\begin{array}{c}29,8 \\
(27,72-31,88)\end{array}$ & $\begin{array}{c}37,8 \\
(35,60-40,00)\end{array}$ & $\begin{array}{c}37,0 \\
(34,81-39,19)\end{array}$ & $\begin{array}{c}39,1 \\
(36,89-41,31)\end{array}$ & $\begin{array}{c}35,3 \\
(33,13-37,47)\end{array}$ & 0,027 \\
\hline 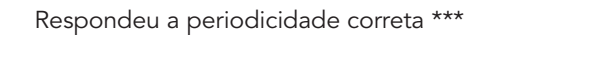 & $\begin{array}{c}61,4 \\
(59,19-63,61)\end{array}$ & $\begin{array}{c}68,6 \\
(66,49-70,71)\end{array}$ & $\begin{array}{c}67,2 \\
(65,07-69,33)\end{array}$ & $\begin{array}{c}87,0 \\
(85,47-88,53)\end{array}$ & $\begin{array}{c}68,0 \\
(65,88-70,12)\end{array}$ & 0,008 \\
\hline
\end{tabular}

* Teste de qui-quadrado $(p<0,05)$;

** Percentuais correspondem aos que afirmaram conhecer o PNVITA (43,5\% dos entrevistados);

*** Percentuais correspondem aos que afirmaram conhecer a periodicidade de suplementação (35,3\% dos entrevistados).

cidade recomendada pelo programa (semestral) 10,11 , sendo observada tendência de aumento das proporções deste conhecimento com o aumento da escolaridade (Tabela 1).

\section{Conhecimento acerca da vitamina A}

Quanto ao conhecimento acerca da vitamina A identificou-se associação direta entre a escolaridade dos responsáveis e a percepção correta sobre a importância desta vitamina, os agravos decorrentes de sua deficiência e seus alimentosfonte. Evidenciou-se tendência de aumento nas proporções de conhecimento com o aumento dos anos de estudo (Tabela 2).

Entre os que afirmaram corretamente a importância da vitamina A, predominaram referências à sua contribuição no crescimento e desenvolvimento da criança $(38,4 \%)$, na promoção da saúde da visão $(28,4 \%)$ e no aumento da imunidade $(22,9 \%)$. Outras características menos expressivas nas referências dos entrevistados foram: complemento nutricional $(7,7 \%)$, saúde da pele/cabelo/unhas $(2,1 \%)$ e proteção contra diarréia e infecções $(0,6 \%)$.

Quanto aos agravos referidos de forma coerente pelos entrevistados, tem-se: cegueira $(42,8 \%)$, imunidade baixa $(35,2 \%)$, anemia $(23,1 \%)$, distúrbios no crescimento e desenvolvimento $(14,3 \%)$ e pele ressecada $(8,8 \%)$. Já os problemas citados de forma incoerente foram: resfriado/gripe $(50,7 \%)$, paralisia $(20,9 \%)$, febre (6\%) e desidratação (3\%).
Ao serem questionados a respeito das fontes de vitamina $\mathrm{A}$, os responsáveis citaram corretamente alimentos como a cenoura, o leite e seus derivados, as folhas verde-escuras, o jerimum (abóbora) e as vísceras (em especial o fígado). No entanto, fontes inespecíficas desse micronutriente, como a laranja e a cenoura, também foram referidas.

\section{Acesso ao PNVITA}

No sentido de avaliar o acesso às megadoses de vitamina $\mathrm{A}$, os entrevistados foram questionados se suas crianças tomam ou já tomaram na USF algum líquido ou cápsula de vitamina. A maioria (62,1\%; IC95\%: 59,90-64,30) referiu positivamente, tendo sido observada maior proporção no grupo de menor escolaridade (0-4 anos) e proporções similares nos demais grupos. A vitamina A foi identificada como a vitamina administrada na USF por mais de cinqüenta por cento $(50,4 \%$; IC95\%: 48,13-52,67) dos entrevistados, com diferença entre as proporções e associação direta com a escolaridade (Tabela 3 ).

No intuito de identificar a existência de mecanismos de garantia do direito ao acesso às megadoses de vitamina $\mathrm{A}$, perguntou-se aos responsáveis a qual órgão recorreriam no caso de falta de cápsulas nas USF. Menos da metade dos entrevistados (40,8\%; IC95\%: 38,57-43,03) respondeu positivamente a essa questão, sendo observada tendência de maior identificação desses órgãos nos grupos com maior escolaridade, com dife- 
Tabela 2

Conhecimento dos responsáveis acerca da vitamina A. Município de Cabedelo, Paraíba, Brasil, 2008.

\begin{tabular}{|c|c|c|c|c|c|c|}
\hline \multirow[t]{3}{*}{ Questões } & \multicolumn{4}{|c|}{ Escolaridade (anos de estudo) } & \multirow[t]{2}{*}{ Total } & \multirow{3}{*}{$\begin{array}{l}\text { Valor } \\
\text { de } p \text { * }\end{array}$} \\
\hline & $0-4$ & $5-8$ & $9-11$ & 12 e mais & & \\
\hline & $\%(I C 95 \%)$ & $\%(I C 95 \%)$ & $\%(I C 95 \%)$ & $\%(I C 95 \%)$ & $\%$ (IC95\%) & \\
\hline \multirow{2}{*}{$\begin{array}{l}\text { Referiu corretamente alguma importância da } \\
\text { vitamina A }\end{array}$} & 16,6 & 20,6 & 24,2 & 40,6 & 22,2 & $<0,001$ \\
\hline & $(14,91-18,29)$ & $(18,76-22,44)$ & $(22,26-26,14)$ & $(38,37-42,83)$ & $(20,31-24,09)$ & \\
\hline \multirow{2}{*}{$\begin{array}{l}\text { Referiu conhecer os agravos da deficiência } \\
\text { de vitamina A }\end{array}$} & 28,0 & 29,3 & 30,4 & 27,5 & 29,3 & 0,819 \\
\hline & $(25,96-30,04)$ & $(27,23-31,37)$ & $(28,31-32,49)$ & $(25,47-29,53)$ & $(27,23-31,37)$ & \\
\hline \multirow{2}{*}{$\begin{array}{l}\text { Referiu corretamente algum agravo da deficiência } \\
\text { de vitamina } A \text { ** }\end{array}$} & 29,4 & 35,4 & 63,9 & 68,4 & 46,6 & $<0,001$ \\
\hline & $(27,33-31,47)$ & $(33,23-37,57)$ & $(61,72-66,08)$ & $(66,29-70,51)$ & $(44,34-48,86)$ & \\
\hline \multirow[t]{2}{*}{ Referiu conhecer os alimentos-fonte de vitamina A } & 50,6 & 36,0 & 38,3 & 40,6 & 40,7 & $<0,001$ \\
\hline & $(48,33-52,87)$ & $(33,82-38,18)$ & $(36,09-40,51)$ & $(38,37-42,83)$ & $(38,47-42,93)$ & \\
\hline Referiu corretamente alimentos-fonte de & 22,0 & 29,7 & 41,2 & 62,5 & 33,4 & $<0,001$ \\
\hline vitamina $A * \star \star$ & $(20,12-23,88)$ & $(27,63-31,77)$ & $(38,97-43,43)$ & $(60,30-64,70)$ & $(31,26-35,54)$ & \\
\hline
\end{tabular}

* Teste de qui-quadrado $(p<0,05)$;

** Percentuais correspondem aos que afirmaram saber algum agravo decorrente da carência de vitamina A ( $29,3 \%$ dos entrevistados);

*** Percentuais correspondem aos que afirmaram conhecer os alimentos-fonte de vitamina A (40,7\% dos entrevistados).

Tabela 3

Acesso ao Programa Nacional de Suplementação de Vitamina A (PNVITA) a partir da percepção dos responsáveis por crianças menores de cinco anos. Município de Cabedelo, Paraíba, Brasil, 2008.

\begin{tabular}{|c|c|c|c|c|c|c|}
\hline \multirow[t]{3}{*}{ Questões } & \multicolumn{5}{|c|}{ Escolaridade (anos de estudo) } & \multirow{3}{*}{$\begin{array}{l}\text { Valor } \\
\text { de } p \text { * }\end{array}$} \\
\hline & $0-4$ & $5-8$ & $9-11$ & 12 e mais & Total & \\
\hline & $\%(I C 95 \%)$ & $\%$ (IC95\%) & $\%$ (IC95\%) & $\%$ (IC95\%) & $\%$ (IC95\%) & \\
\hline Referiu que a criança toma ou já tomou vitamina na USF & $\begin{array}{c}68,0 \\
(65,88-70,12)\end{array}$ & $\begin{array}{c}60,0 \\
(57,78-62,22)\end{array}$ & $\begin{array}{c}59,2 \\
(56,97-61,43)\end{array}$ & $\begin{array}{c}62,8 \\
(60,61-64,99)\end{array}$ & $\begin{array}{c}62,1 \\
(59,90-64,30)\end{array}$ & $<0,001$ \\
\hline $\begin{array}{l}\text { Referiu que a criança toma ou já tomou vitamina A } \\
\text { na USF ** }\end{array}$ & $\begin{array}{c}38,5 \\
(36,29-40,71)\end{array}$ & $\begin{array}{c}49,6 \\
(47,33-51,87)\end{array}$ & $\begin{array}{c}56,7 \\
(54,45-58,95)\end{array}$ & $\begin{array}{c}74,4 \\
(72,42-76,38)\end{array}$ & $\begin{array}{c}50,4 \\
(48,13-52,67)\end{array}$ & $<0,001$ \\
\hline Referiu já ter faltado cápsula de vitamina A na USF & $\begin{array}{c}15,3 \\
(13,67-16,93)\end{array}$ & $\begin{array}{c}14,0 \\
(12,43-15,57)\end{array}$ & $\begin{array}{c}13,9 \\
(12,33-15,47)\end{array}$ & $\begin{array}{c}8,1 \\
(6,86-9,34)\end{array}$ & $\begin{array}{c}13,8 \\
(12,23-15,37)\end{array}$ & 0,136 \\
\hline $\begin{array}{l}\text { Referiu saber a quem reclamar se faltar cápsula } \\
\text { de vitamina A na USF }\end{array}$ & $\begin{array}{c}34,2 \\
(32,05-36,35)\end{array}$ & $\begin{array}{c}38,7 \\
(36,49-40,91)\end{array}$ & $\begin{array}{c}44,8 \\
(42,54-47,06)\end{array}$ & $\begin{array}{c}49,3 \\
(47,03-51,57)\end{array}$ & $\begin{array}{c}40,8 \\
(38,57-43,03)\end{array}$ & $<0,001$ \\
\hline $\begin{array}{l}\text { Referiu ter recebido orientação sobre alimentação e } \\
\text { nutrição na USF }\end{array}$ & $\begin{array}{c}64,3 \\
(62,13-66,47)\end{array}$ & $\begin{array}{c}64,5 \\
(62,33-66,67)\end{array}$ & $\begin{array}{c}64,6 \\
(62,43-66,77)\end{array}$ & $\begin{array}{c}28,5 \\
(26,45-30,55)\end{array}$ & $\begin{array}{c}61,8 \\
(59,59-64,01)\end{array}$ & $<0,001$ \\
\hline
\end{tabular}

USF: unidade de saúde da família.

* Teste de qui-quadrado $(p<0,05)$;

** Percentuais correspondem aos que afirmaram que a criança toma ou já tomou alguma vitamina na USF (62,1\% dos entrevistados).

rença entre as proporções (Tabela 3). Os órgãos mais citados foram a Secretaria de Saúde $(57,3 \%)$ e a própria USF (30,8\%).

A maioria dos responsáveis $(61,8 \%$; IC95\%: 59,59-64,01) referiu já ter recebido alguma orientação sobre alimentação e nutrição na USF. O grupo que referiu em menor proporção ter rece- bido essas orientações foi aquele com 12 anos e mais de estudo (Tabela 3). Segundo os entrevistados, os temas abordados em geral nas atividades educativas são: orientações gerais para uma alimentação saudável (33\%); alimentação saudável para crianças $(23,6 \%)$; alimentação nas diversas patologias $(9,1 \%)$; aleitamento materno $(7,8 \%)$; 
alimentação saudável para gestantes (3\%); vitamina A $(0,9 \%)$; manipulação de alimentos/culinária $(0,4 \%)$.

\section{Cobertura de vitamina $\mathrm{A}$}

Ao verificar as doses de vitamina A registradas pelas USF nos cartões de vacinação das crianças menores de cinco anos, observou-se que os maiores percentuais de cobertura do PNVITA aparecem no grupo de crianças de 6 a 17 meses de idade. No entanto, mesmo nessa faixa etária, a cobertura apresenta-se abaixo da meta recomendada pelo programa, agravando-se ainda mais com o avançar da idade da criança. Além disso, mais de $30 \%$ das crianças na faixa etária de 6 a 11 meses não receberam nenhuma megadose de vitamina $A$, apesar de a coleta de dados ter sido realizada na segunda etapa da Campanha de Multivacinação de 2008. Observa-se, ainda, que a partir dos 18 meses de idade a cobertura situa-se abaixo de trinta por cento, sendo as piores coberturas presentes a partir de 30 meses de idade (percentuais inferiores a 11\%) (Tabela 4).

\section{Discussão}

Os resultados do estudo evidenciaram baixos percentuais de conhecimento dos responsáveis pelas crianças público-alvo do PNVITA acerca deste, bem como sobre o micronutriente.

Esse desconhecimento perpassou desde a não identificação da existência do programa até aspectos específicos relacionados à importância da vitamina A e aos agravos que podem ocorrer com a deficiência desta vitamina.
Muitos responsáveis reconheceram a distribuição de cápsulas vitamínicas na USF sem, contudo, identificá-las como sendo as megadoses de vitamina $\mathrm{A}$, especialmente entre os entrevistados com menos anos de estudo (0-4 anos). Além disso, não visualizam essa ação como componente de um programa institucionalizado, o qual se configura como uma estratégia de segurança alimentar e nutricional desenvolvida no âmbito da gestão das políticas de alimentação e nutrição. No estudo de Martins et al. 16, 49,7\% dos responsáveis identificaram a administração de vitamina A à sua criança.

Esse fato é preocupante para a sustentabilidade do programa, já que não permite a apropriação deste por parte da população, que não o vislumbra como um direito social e de saúde. Além disso, a falta de conhecimento sobre a vitamina A pode interferir diretamente na adesão e aderência às ações do programa, refletindo baixos percentuais de cobertura - que inclusive foram observados neste estudo.

Verificou-se, ainda, que a escolaridade exerce influência positiva no conhecimento dos responsáveis, tendo em vista maiores proporções de ciência das questões abordadas neste trabalho nos grupos com mais anos de estudo. Nesses grupos, percebeu-se um maior conhecimento no tocante à vitamina $\mathrm{A}$, figurado na coerência das respostas acerca da importância, dos agravos decorrentes da deficiência e dos alimentos-fonte deste micronutriente. No entanto, percebeu-se um maior desconhecimento desse grupo, quando comparado aos demais, acerca das ações relacionadas ao programa de suplementação dessa vitamina, que pode ser justificado pela baixa participação desses nas ações educativas referentes ao progra-

Tabela 4

Cobertura da distribuição de megadoses de vitamina A às crianças acompanhadas por unidades de saúde da família (USF).

Município de Cabedelo, Paraíba, Brasil, 2008.

\begin{tabular}{|c|c|c|c|c|c|c|c|c|c|c|c|}
\hline \multirow{2}{*}{$\begin{array}{l}\text { Idade da } \\
\text { criança } \\
\text { (meses) }\end{array}$} & \multirow{2}{*}{$\begin{array}{c}\text { Doses } \\
\text { recomen- } \\
\text { dadas }\end{array}$} & \multicolumn{10}{|c|}{ Doses recebidas (\%) } \\
\hline & & 0,0 & 1,0 & 2,0 & 3,0 & 4,0 & 5,0 & 6,0 & 7,0 & 8,0 & 9,0 \\
\hline $6-11$ & 1,0 & 30,9 & 66,6 & 2,5 & & & & & & & \\
\hline $12-17$ & 2,0 & 28,1 & 22,8 & 43,8 & 1,5 & 3,8 & & & & & \\
\hline $18-23$ & 3,0 & 9,7 & 19,6 & 39,5 & 27,9 & 3,4 & & & & & \\
\hline $24-29$ & 4,0 & 17,0 & 17,9 & 18,8 & 34,2 & 12,1 & & & & & \\
\hline $30-35$ & 5,0 & 11,8 & 4,7 & 20,1 & 25,3 & 28,0 & 8,6 & 1,5 & & & \\
\hline $36-41$ & 6,0 & 22,5 & 6,4 & 9,5 & 11,1 & 18,6 & 21,6 & 10,3 & & & \\
\hline $42-47$ & 7,0 & 14,3 & 2,3 & 13,7 & 13,4 & 13,3 & 23,0 & 16,5 & 2,3 & 1,1 & \\
\hline $48-53$ & 8,0 & 10,2 & 2,9 & 8,3 & 10,0 & 13,9 & 17,2 & 18,9 & 16,0 & 2,7 & \\
\hline $54-59$ & 9,0 & 7,2 & 2,9 & 10,3 & 8,7 & 12,4 & 8,5 & 12,9 & 22,8 & 9,5 & 4,8 \\
\hline
\end{tabular}


ma. Esse último fato evidencia que a aquisição das informações acerca da vitamina A por parte desse grupo se deu extramuros da USF.

Os intervalos de confiança permitiram identificar um comportamento diferenciado no grupo de entrevistados com 12 e mais anos de estudo quando comparado aos demais grupos de escolaridade, visto que este grupo, por vezes, não mantinha a tendência observada. Esse resultado pode ser explicado pelo fato de o grupo com maior escolaridade (12 e mais anos de estudo) não ser público-alvo da Estratégia Saúde da Família (ESF), visto que esta é voltada aos grupos em maior vulnerabilidade social.

Ribeiro et al. 21, ao analisar os dados da Pesquisa Nacional por Amostra de Domicílios (PNAD) do ano de 2003, identificaram que a maior parte da população atendida pelo Programa Saúde da Família (PSF) apresenta menos de três anos de estudo, enquanto que a população atendida pelo sistema privado apresenta alta escolaridade (11 e mais anos de estudo). Além disso, pesquisas apontam que o grau de insegurança alimentar de uma população é significativamente maior nos grupos com menor escolaridade 22,23.

No que diz respeito aos grupos com menos anos de estudo, percebeu-se que apesar de terem afirmado em maior proporção a participação nas ações educativas em alimentação e nutrição, bem como de terem identificado as ações do programa em maior proporção que os demais grupos, desconhecem a importância da vitamina $\mathrm{A}$, o que pode ser conseqüência dos baixos percentuais de referência à ocorrência de ações de informação acerca desta vitamina e de seu programa de suplementação, denotando a insuficiente abordagem educativa sobre este tema dentro das ações de promoção à saúde desenvolvidas nas USF.

Assim, como visto nos resultados que a maioria dos entrevistados possui baixo grau de instrução (56,5\% tinham até o Ensino Fundamental), devem ser pensadas estratégias de apreensão do conhecimento que favoreçam o diálogo, a troca, a transdisciplinaridade entre os distintos saberes formais e não-formais, os quais contribuam para as ações de promoção de saúde em nível individual e coletivo 24 .

O desconhecimento acerca das fontes alimentares de vitamina A e dos benefícios de sua ingestão também constitui um obstáculo à segurança alimentar e nutricional da população, visto que pode contribuir para a baixa inclusão destes alimentos nas refeições das crianças. Além disso, considerando a maior proporção de mães na população deste estudo, esperavase referências ao leite materno como fonte de vitamina $\mathrm{A}$, tendo em vista a extensão do pro- grama às puérperas desde 200125 . No entanto, nenhum entrevistado citou esse alimento como fonte de vitamina $A$.

A literatura especializada aponta que a deficiência de vitamina A tem maior associação com o consumo inadequado de fontes deste nutriente do que com fatores econômicos 26,27. Nesse sentido, o aumento do consumo desses alimentos apresenta-se como a principal estratégia, a médio e longo prazos, no combate à hipovitaminose A em nível mundial, tendo as mulheres papel primordial no estímulo à incorporação de hábitos alimentares saudáveis na família e comunidade, visto que em geral controlam as práticas alimentares familiares, principalmente quando da introdução de alimentos no período de desmame e na alimentação das crianças em idade pré-escolar 28,29.

A escolha dos alimentos a serem consumidos é um processo complexo, que pode ser influenciado por fatores que perpassam o acesso aos alimentos e o conhecimento em alimentação e nutrição, como por exemplo, fatores culturais e hábitos alimentares da população. Esse conjunto de situações indica a importância da educação nutricional e seu relevante papel no estabelecimento de hábitos alimentares adequados, como parte das ações de controle das deficiências nutricionais, em especial nas regiões consideradas de risco 30.

Aliado à intensificação das ações educativas, devem ser desenvolvidas e/ou fortalecidas estratégias de articulação do PNVITA com outros programas de segurança alimentar e nutricional, como o Programa de Aquisição de Alimentos, o Programa Bolsa Família e o Programa Nacional de Alimentação Escolar, no intuito de promover o acesso e consumo de alimentos-fonte de vitamina A, assim como a disseminação de informações a respeito destes alimentos.

Estudo realizado por Martins et al. 16 identificou que a maioria $(74,6 \%)$ dos responsáveis pelas crianças público-alvo do PNVITA relatou não possuir nenhum tipo de conhecimento sobre essa vitamina, em que apenas 10,9\% relataram ter obtido informações sobre a mesma, principalmente nas unidades de saúde. Nesse mesmo estudo, quando questionados acerca do tipo de informação obtida sobre a vitamina A, 47,3\% relataram que ela ajuda na nutrição da criança, no entanto alegações sem respaldo científico também foram obtidas, tais como o combate à gripe.

Na literatura científica são escassos os estudos que avaliaram o conhecimento dos responsáveis pelas crianças participantes do PNVITA acerca deste. Entretanto, são encontradas avaliações de outros programas e políticas públicas na área de alimentação e nutrição, dentre os quais 
pode ser citado o de Santos et al. 31. Esses autores, ao avaliarem o Programa de Alimentação do Trabalhador no interior do Estado da Bahia, observaram que apenas $15 \%$ dos indivíduos participantes deste programa declararam ter algum tipo de conhecimento sobre o mesmo, indicando sua insuficiente divulgação entre os potenciais interessados, apesar de tratar-se de um programa com tantos anos de existência.

No que tange à cobertura do Programa de Vitamina A, os resultados deste estudo corroboram com dados divulgados no sítio eletrônico da CGPAN sobre a cobertura nacional do programa para o ano de 2008 (ano deste estudo), os quais mostram que a mesma foi de $85,5 \%$ para crianças entre 6 e 11 meses; e $43 \%$ e 26,8\% para a primeira e segunda doses em crianças de 12 a 59 meses, respectivamente 32 .

Segundo Martins et al. 8, no período de 1994 (ano de instituição da Portaria que regulamentou o programa) a 2003, a cobertura do PNVITA oscilou entre $28 \%$ e $72 \%$. Observou-se ainda, assim como os dados encontrados neste trabalho, que a cobertura da distribuição de megadoses de vitamina A em crianças de 6 a 11 meses é, geralmente, mais elevada do que aquela observada para crianças de 12 a 59 meses de idade. Acredita-se que esse fato deve-se aos contatos rotineiros, durante essa faixa etária, com os serviços primários de atenção à saúde, como a imunização e a puericultura (acompanhamento do crescimento e desenvolvimento).

Considerando que um dos momentos de contato das crianças com os serviços de saúde que poderia elevar a cobertura do PNVITA seria aquele representado pela vacinação, esperava-se maiores percentuais de cobertura do programa no presente estudo, tendo em vista a realização da coleta de dados na segunda etapa da Campanha de Multivacinação 2008. No entanto, 19,2\% das crianças na faixa etária para receber a suplementação de vitamina A não havia recebido nenhuma dose deste micronutriente.

Diante do exposto, percebe-se que as baixas coberturas do programa podem ser determinadas pela falta de conhecimento dos responsáveis sobre a relevância dessa ação, tornando-se um prejuízo à segurança alimentar e nutricional da população, em especial aquela de maior vulnerabilidade social (contexto socioeconômico desfavorável). Outro fato que pode elucidar a cobertura observada neste estudo é a ausência de controle na administração das doses de vitamina A às crianças, cujo registro era realizado em mapas diários que não permitiam a identificação da criança que recebia a megadose, mas apenas a quantidade de doses administradas diariamente.
Nesse sentido, devem ser potencializadas ações que visem à ampliação da cobertura em todas as faixas etárias, como a intensificação da busca ativa das crianças público-alvo do programa por parte dos profissionais inseridos nas equipes de saúde da família, em especial o agente comunitário de saúde (ACS), pelo seu contato rotineiro com as famílias. Além disso, a forma de controle do registro das doses administradas deve ser reestruturada, no intuito de facilitar a identificação das crianças que já receberam as megadoses, bem como daquelas que ainda estão por receber, contribuindo assim para uma melhor captação do público-alvo do programa.

Além da abordagem aos responsáveis por crianças menores de cinco anos, neste trabalho também foram entrevistados outros atores sociais envolvidos na operacionalização do Programa no município, como os profissionais inseridos nas equipes de saúde da família e gestores responsáveis pelo programa no município (Coordenação de Alimentação e Nutrição e Coordenação da Atenção Básica). Uma análise preliminar desses dados evidenciou fragilidade no conhecimento acerca da vitamina A e do programa, além da ausência de ciclos de capacitação sobre esse tema.

Uma limitação deste estudo foi a não inclusão das nutrizes no pós-parto imediato. Assim, recomenda-se aos futuros pesquisadores desse tema que seja investigada a percepção desses atores sociais, a fim de identificar a cobertura do programa nessa população, além das ações direcionadas a esse público.

\section{Considerações finais}

Diante do exposto, faz-se necessária a intensificação de ações educativas relacionadas ao PNVITA, no intuito de orientar e sensibilizar os responsáveis quanto à importância desta vitamina, de sua suplementação e da ingestão de alimentosfonte deste nutriente para a saúde da criança. Essas ações devem ser planejadas levando-se em conta o perfil sociodemográfico da população, em especial aquelas que se encontram em maior grau de vulnerabilidade social, considerando-se o grau de escolaridade do público a que se destinam e a metodologia mais apropriada de repasse de informações, com o objetivo de facilitar o encontro entre o sujeito que aprende e o conhecimento a ser aprendido.

Os resultados deste estudo foram apresentados e discutidos em oficina com a participação do coordenador da área de alimentação e nutrição e nutricionistas inseridos na ESF do Município de Cabedelo, além de representantes da 
Gerência Operacional de Alimentação e Nutrição da Secretaria de Saúde do Estado da Paraíba. Nessa oficina foi produzida uma proposta de plano de ação para aperfeiçoamento do Programa. A primeira estratégia posta em prática no Município de Cabedelo foi a instituição, em todas as USF, de um livro padronizado para acompanhamento das doses administradas à criança da área de abrangência. A idéia é intensificar a identificação das crianças com doses descobertas e fazer busca ativa por intermédio do ACS, facilitando também as orientações individuais.

Espera-se que este processo avaliativo norteie ações de reorientação do programa não apenas em Cabedelo, mas também em âmbito estadual e nacional, com o objetivo de promover a gestão participativa do programa e a realização de ações de educação continuada direcionada aos atores sociais nele envolvidos.

\section{Resumo}

Esta pesquisa teve o propósito de avaliar o Programa Nacional de Suplementação de Vitamina A em um município da Região Nordeste do Brasil, com a participação de responsáveis por crianças menores de cinco anos de idade. Trata-se de um estudo transversal, de base populacional, com abordagens qualitativa e quantitativa. Foram realizadas 657 entrevistas estruturadas e análise do registro das doses de vitamina $A$ nos cartões de vacinação das crianças. Observou-se que a população tem acesso às megadoses de vitamina A. No entanto, percebeu-se uma fragilidade no conhecimento acerca do programa e da vitamina A, especialmente entre a população de menor escolaridade. Observou-se ainda que a cobertura do programa está aquém da meta recomendada pelo Ministério da Saúde, sobretudo a partir dos 18 meses de idade. Diante do exposto, é necessário intensificar as ações educativas relacionadas ao programa, no intuito de orientar e sensibilizar os responsáveis quanto à importância da vitamina A, de sua suplementação e da ingestão de alimentos-fonte deste nutriente para a saúde da criança.

Vitamina A; Programas e Políticas de Alimentação e Nutrição; Saúde da Criança

\section{Colaboradores}

E. R. Almeida e A. T. Carvalho participaram do delineamento metodológico, elaboração dos instrumentos, coleta, análise e interpretação dos dados, concepção e redação deste artigo. E. A. F. Nilson, J. G. Coutinho e J. A. Ubarana participaram na concepção do projeto, no delineamento metodológico, elaboração dos instrumentos, estruturação e análise do banco de dados, bem como na aprovação final da versão a ser publicada.

\section{Agradecimentos}

Agradecemos à Secretaria de Saúde do Município de Cabedelo, Paraíba, por permitir a realização deste trabalho; a todos os sujeitos da pesquisa, que aceitaram ser entrevistados; e aos alunos de graduação em Nutrição da Universidade Federal da Paraíba que atuaram como entrevistadores. Aos professores Rodrigo Vianna e Cristina Raposo pelas contribuições na análise estatística dos dados. Ao professor Alcides Diniz pelas contribuições na primeira versão do artigo. À Coordenação Geral da Política de Alimentação e Nutrição e à Organização das Nações Unidas para Alimentação e Agricultura pelo apoio logístico, técnico e financeiro. 


\section{Referências}

1. World Health Organization. Global prevalence of vitamin A deficiency in populations at risk 19952005. Geneva: World Health Organization; 2009.

2. Wolf G, Phil D. A historic note on the mode of administration of vitamin A for the cure of night blindness. Am J Clin Nutr 1978; 31:290-2.

3. Santos LMP, Batista-Filho M, Diniz AS. Epidemiologia da carência de vitamina A no Nordeste do Brasil. Bol Oficina Sanit Panam 1996; 120:525-37.

4. Fawzi WW, Chalmers TC, Herrera MG, Mosteller F. Vitamin A supplementation and child mortality: a meta-analysis. JAMA 1993; 269:898-903.

5. Beaton GH, Martorell R, Aronson KA, Edmonston B, McCabe G, Ross AC, et al. La suplementación con vitamina A y la morbilidad y mortalidad infantil en los países en desarrollo. Bol Oficina Sanit Panam 1994; 117:506-18.

6. Assis AMO, Barreto ML. Suplementação com vitamina A: impacto na morbidade e efeitos adversos. Rev Bras Epidemiol 2002; 5:84-92.

7. Oliveira JM, Rondó PHC. Evidências do impacto da suplementação de vitamina A no grupo materno infantil. Cad Saúde Pública 2007; 23:2565-75.

8. Martins MC, Oliveira YP, Coitinho DC, Santos LMP Panorama das ações de controle da deficiência de vitamina "A" no Brasil. Rev Nutr 2007; 20:5-18.

9. Brasil. Portaria ${ }^{\circ}$. 2.160, de 23 de dezembro de 1994. Cria, no Instituto Nacional de Alimentação e Nutrição, o Programa Nacional de Controle das Deficiências de Vitamina A e dá outras providências. Diário Oficial da União 1994; $30 \mathrm{dez}$.

10. Brasil. Portaria n ${ }^{\circ} .729$, de 13 de maio de 2005. Institui o Programa Nacional de Suplementação de Vitamina A e dá outras providências. Diário Oficial da União 2005; 16 mai.

11. Secretaria de Atenção à Saúde, Ministério da Saúde. Vitamina A Mais: Programa Nacional de Suplementação de Vitamina A - condutas gerais. Brasília: Ministério da Saúde; 2004. (Série A. Normas e Manuais Técnicos).

12. Ramalho A, Padilha P, Saunders C. Análise crítica de estudos brasileiros sobre deficiência de vitamina A no grupo materno-infantil. Rev Paul Pediatr 2008; 26:392-9.

13. Saunders C, Ramalho RA, Lima AP, Gomes MM, Campos LF, Silva BA, et al. Association between gestational night blindness and serum retinol in mother/newborn pairs in the city of Rio de Janeiro, Brazil. Nutrition 2005; 21:456-61.

14. Sarni RS, Kochi C, Ramalho RA, Schoeps DO, Sato $\mathrm{K}$, Mattoso L, et al. Impact of vitamin A megadose supplementation on the anthropometry of children and adolescents with non-hormonal statural deficit: a double-blind and randomized clinical study. Int J Vitam Nutr Res 2003; 73:303-11.

15. West Jr. KP, Katz J, Khatry SK, LeClerq SC, Pradhan EK, Shrestha SR, et al. Double blind, cluster randomised trial of low dose supplementation with vitamin A or beta carotene on mortality related to pregnancy in Nepal. The NNIPS-2 Study Group. BMJ 1999; 318:570-5.
16. Martins MC, Santos LMP, Santos SMC, Araújo MPN, Lima AMP, Santana LAA. Avaliação de políticas públicas de segurança alimentar e combate à fome no período de 1995-2002. 3 - O Programa Nacional de Controle da Deficiência de Vitamina A. Cad Saúde Pública 2007; 23:2081-93.

17. Marinho A, Façanha LO. Programas sociais: efetividade, eficiência e eficácia como dimensões operacionais da avaliação. Brasília: Instituto de Pesquisa Econômica Aplicada; 2001. (Texto para Discussão, 787)

18. Minayo MCS, Cruz Neto O. Triangulación de métodos en la evaluación de programas y servicios de salud. In: Bronfman M, Castro R, editores. Salud, cambio social y política: perspectivas desde América Latina. Cor del Valle: Edamex; 1999. p. 65-80.

19. Coordenação de Acompanhamento e Avaliação, Departamento de Atenção Básica, Secretaria de Atenção à Saúde, Ministério da Saúde. Avaliação da atenção básica em saúde: caminhos da institucionalização. Brasília: Ministério da Saúde; 2005.

20. Semba RD, Bloem MW. The anemia of vitamin A deficiency: epidemiology and pathogenesis. Eur J Clin Nutr 2002; 56:271-81.

21. Ribeiro MCSA, Barata RB, Almeida MF, Silva ZP. Perfil sociodemográfico e padrão de utilização de serviços de saúde para usuários e não-usuários do SUS - PNAD 2003. Ciênc Saúde Coletiva 2006; 11:1011-22

22. Marin-León L, Segall-Corrêa AM, Panigassi G, Maranha LK, Sampaio MFA, Pérez-Escamilla R. A percepção de insegurança alimentar em famílias com idosos em Campinas, São Paulo, Brasil. Cad Saúde Pública 2005; 21:1433-40.

23. Fávaro T, Ribas DLB, Zorzatto JR, Segall-Corrêa AM, Panigassi G. Segurança alimentar em famílias indígenas Teréna, Mato Grosso do Sul, Brasil. Cad Saúde Pública 2007; 23:785-93.

24. Machado MFAS, Monteiro EMLM, Queiroz DT, Vieira NFC, Barroso MGT. Integralidade, formação de saúde, educação em saúde e as propostas do SUS: uma revisão conceitual. Ciênc Saúde Coletiva 2007; $12: 335-42$.

25. Secretaria de Políticas de Saúde, Ministério da Saúde. Projeto suplementação de mega dose de vitamina A no pós-parto imediato nas maternidades/hospitais. Brasília: Ministério da Saúde; 2002.

26. Brunken GS, Flores H. Consumption of vitamin A rich foods. Xerophtalmia Club Bulletin 1993; 54:3-4.

27. Coelho CSP, Ramalho RA, Accioly E. O inquérito dietético na avaliação do estado nutricional de vitamina A em gestantes. Ars Cvrandi 1995; 6:44-60.

28. Ramalho RA, Anjos LA, Flores H. Hipovitaminose A em recém-nascidos em duas maternidades públicas no Rio de Janeiro, Brasil. Cad Saúde Pública 1998; 14:821-7.

29. Ramalho RA, Flores H, Accioly E, Saunders C. Associação entre deficiência de vitamina A e situação sociodemográfica de mães e recém-nascidos. Rev Assoc Med Bras 2006; 52:170-5. 
30. Pereira JA, Paiva AA, Bergamaschi DP, Rondó PHC, Oliveira GC, Lopes IBM, et al. Concentrações de retinol e de beta-caroteno séricos e perfil nutricional de crianças em Teresina, Piauí, Brasil. Rev Bras Epidemiol 2008; 11:287-96.

31. Santos LMP, Araújo MPN, Martins MC, Veloso IS Assunção MP, Santos SMC. Avaliação de políticas públicas de segurança alimentar e combate à fome no período 1995-2002. 2 - Programa de Alimentação do Trabalhador. Cad Saúde Pública 2007; 23:1931-45.
32. Secretaria de Atenção à Saúde, Ministério da Saúde. Vitamina A Mais: programa nacional de suplementação de vitamina A. http://dtr2004.saude. gov.br/nutricao/vita_relatorio.php (acessado em 06/Jun/2009).

Recebido em 06/Jul/2009

Versão final reapresentada em 14/Dez/2009

Aprovado em 24/Mar/2010 